\title{
Effects of pipe angular velocity and oven configuration on tube temperature distribution in the radiative heating of PVC pipes
}

\author{
Michael Lucchi ${ }^{1} \cdot$ Marco Lorenzini $^{1}$ (D)
}

Received: 18 September 2017 / Accepted: 26 December 2017 / Published online: 23 January 2018

(C) The Author(s) 2018. This article is an open access publication

\begin{abstract}
Several manufacturing processes in polymer industry aim at obtaining products by deforming preforms or sheets after a heating process. A thorough knowledge of the operating parameters of such heating processes is fundamental to fulfill the often high production requirements with the least energy consumption and to avoid unacceptable defects in the final product. A common example of such an application is the end-forming process of polyvinyl chloride (PVC) tubes, which are enlarged at one end in order to allow pipes connections. The heating phase which comes before the deformation process is usually carried out in ovens equipped with short wave infrared lamps; to ensure uniform heating, pipes rotate with a given angular velocity, which represents a fundamental parameter for the success of the whole manufacturing process. In this work, a transient analysis of the radiative heat exchange between rotating PVC pipes and infrared lamps in an oven for end-forming process has been conducted by means of a finite element model, in order to investigate the influence of cylinder angular velocity on the temperature distribution in the tube. Local view factors have been calculated for different oven configurations and have been expressed as a function of angular velocity, allowing pipe rotation to be simulated as a time-dependent boundary condition, instead of using a moving mesh. Simulations were carried out for different tubes geometries and angular velocities and results were compared with the case of a uniformly irradiated tube in terms of temperature displacement. For a given oven configuration, the results obtained by the numerical model can be used to find a critical angular velocity over which further increase does not lead to appreciable improvements in temperature evenness. The effect of the lamps' relative position was also investigated, showing a significant influence on critical angular velocities obtained. The model realized represents a potential tool to characterize the end-forming process in terms of critical angular velocity, leading to reductions in machine set-up time and product waste due to thermal failure.
\end{abstract}

Keywords Pipes end-forming · Radative heating · Finite-element modeling

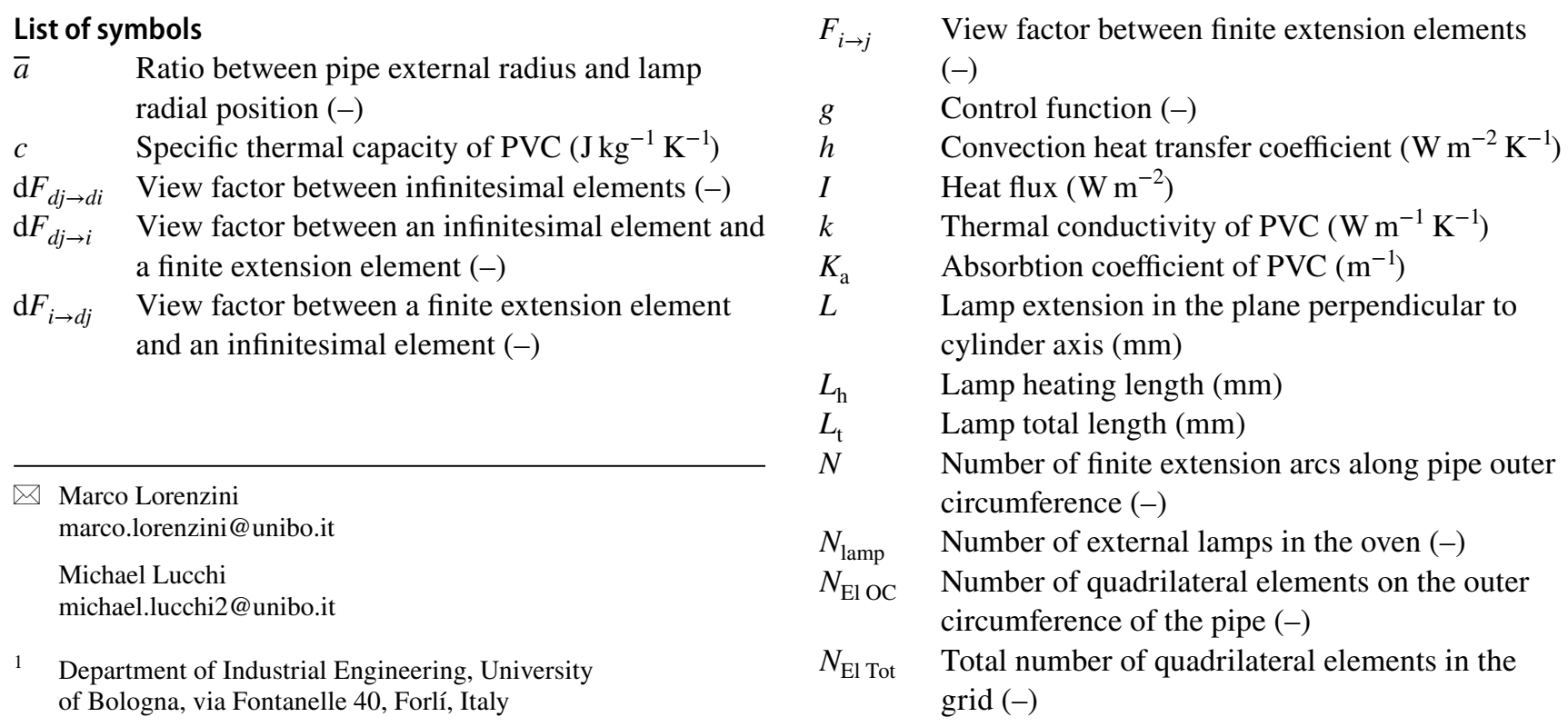


$p \quad$ Lamp facing period (s)

$P_{\mathrm{r}} \quad$ Lamp rated power $(\mathrm{W})$

$q_{\mathrm{g}}^{\prime \prime \prime} \quad$ Heat generation term $\left(\mathrm{W} \mathrm{m}^{-3}\right)$

$r \quad$ Generic radial coordinate $(\mathrm{mm})$

$R_{\mathrm{L}} \quad$ Lamp radial position $(\mathrm{mm})$

$T \quad$ Temperature (K)

$T_{\text {off }} \quad$ Lamps switch off temperature when they are controlled by a traditional pyrometer $(\mathrm{K})$

$T_{\mathrm{s}} \quad$ Softening temperature of PVC (K)

th Pipe thickness (mm)

$x \quad$ Generic coordinate along lamp extension ( $\mathrm{mm})$

$\bar{x} \quad$ Non-dimensional coordinate along lamp extension in a transversal plane perpendicular to cylinder axis (-)

$\bar{x}_{1} \quad$ Non-dimensional coordinate $\bar{x}$ calculated at the first end of the lamp (-)

$\bar{x}_{2} \quad$ Non-dimensional coordinate $\bar{x}$ calculated at the second end of the lamp (-)

\section{Greek letters}

$\alpha \quad$ Thermal diffusivity of PVC $\left(\mathrm{m}^{2} \mathrm{~s}^{-1}\right)$

$\alpha_{\mathrm{L}} \quad$ Lamp angular position (deg)

$\Delta \theta \quad$ Angular extension of finite arcs on pipe outer circumference (rad)

$\Delta T \quad$ Maximum temperature displacement with respect to the case of a perfectly uniform radiation $(\mathrm{K})$

$\Delta T_{\text {eval }} \quad$ Representative maximum temperature displacement to be compared with the maximum allowable one (K)

$\Delta T_{\max } \quad$ Maximum allowable temperature displacement in the heating stage of the end-forming process (K)

$\varepsilon \quad$ Emissivity of PVC (-)

$\theta \quad$ Angular position along pipe outer circumference (rad)

$\theta_{\text {control }}$ Angle between the normal to cylinder outer surface at a point $M$ and the line joining $M$ to a generic point $P$ on the $i$ th lamp (rad)

$\rho \quad$ Density of PVC $\left(\mathrm{kg} \mathrm{m}^{-3}\right)$

$\sigma \quad$ Stefan-Boltzmann constant $\left(\mathrm{W} \mathrm{m}^{-2} \mathrm{~K}^{-4}\right)$

$\tau \quad$ Time (s)

$\omega \quad$ Pipe angular velocity $\left(\mathrm{rad} \mathrm{s}^{-1}\right)$

\section{Subscripts}

di Infinitesimal element on a lamp

$d j \quad$ Infinitesimal element on cylinder outer circumference

$i \quad$ Finite extension lamp

$j \quad$ Finite extension arc on cylinder outer circumference

$r \quad$ Generic radial coordinate

$r_{\mathrm{i}} \quad$ Pipe inner radius $r_{\mathrm{e}} \quad$ Pipe external radius

$r_{\mathrm{m}} \quad$ Pipe mean radius

\section{Introduction}

In the second half of the twentieth century, a rapid increase in the production scales and in the variety and complexity of manufacturing processes characterized the evolution of the polymer industry [1]. In industrial applications like thermoforming, aimed at obtaining products by deforming polymeric preforms or sheets, the study of the thermal stage allows to get a successful forming process and directly affects the mechanical properties of the end product [2]. Moreover, studying the heating stage in the design phase of such processes can lead to a significant increase in the process efficiency, with consequent energy savings and reduced environmental impact. Many authors developed models to analyze the thermal phase of Injection Stretch Blowing Moulding (ISBM) technology. In [3] a finite element model was realized for the thermal analysis of a preform which rotates in front of infrared lamps located along its axis; the same industrial process has been investigated also in [4] using the identical numerical approach of [3]; in the latter case, however, the ray tracing method was adopted to model the heat source term. The thermal phase represents the most critical stage for the end-forming process of polyvinyl chloride (PVC) tubes too, where one end undergoes a deformation process in order to allow piping connection. To soften the material before deformation, the end of the tube is slid into an oven equipped with infrared lamps and a rotation is imposed on the tube so as to ensure uniform heating. Different kinds of defects (e.g. localized burns, wall thinning, excessive shrinkage and loss of elasticity) can occur in the final product due to an uneven temperature distribution within the material, thus causing the pipe to be discarded [5]. Consequently, a fundamental parameter of a successful end-forming process is represented by the tube angular velocity.

The heat transfer from rotating cylinders and between concentric rotating cylinders has been the subject of several research work, as reviewed in [6]: some authors studied the problem of mixed convection on a horizontal rotating cylinder both numerically [7], and experimentally [8]. In [9] an experimental investigation on the convective heat transfer coefficient inside a rotating cylinder with an axial air flow was conducted, while in [10] the transient heat conduction in a rotating cylindrical shell exposed to a time-varying incident heat flux was numerically studied.

In this work, the transient analysis of the radiative heat exchange between rotating PVC pipes and infrared lamps of a oven for end-forming process has been carried out numerically, in order to investigate the influence of cylinder 
angular velocity and oven configuration on the temperature distribution within the tube. The approach adopted was the finite-element method, using COMSOL ${ }^{\circledR}$ as modeling environment; to obtain detailed temperature information in both spatial and time domain, approaches like the lumped parameters modeling, often applied to the transient analysis at system level [11], could not be used.

To avoid a moving mesh, pipe rotation was simulated by assuming a stationary domain subjected to a time-dependent boundary condition. View factors were calculated locally as a function of the geometry and oven configuration and were expressed as a function of angular velocity. The influence of angular velocity on temperature distribution both at the outer surface and within the pipe wall was investigated and compared with the case of uniform heat flux to quantify the temperature unevenness.

The authors began the analysis of the heating stage of the end-forming process in [12], where the influence of parameters such as angular velocity, convective heat transfer coefficients and the presence of inner additional lamps on the temperature unevenness within pipe wall was quantified for a fixed oven configuration, adopting the same approach proposed here. However, only one oven configuration was considered and no information was given about how to choose the most suitable angular velocity values to carry out a proper end-forming process. In this work, two different oven configurations were investigated to analyze the influence of the lamps' angular position on temperature uniformity and the results obtained were subsequently used in order to determine the minimum values of angular velocity which allow to obtain the desired uniformity in temperature distribution. Thus, the aim of this work is to create a design instrument for the end-forming process of polymeric tubes, allowing to determine the most suitable angular velocity values for a successful heating phase. Adding new developments to this work, like implementing a surface temperature control within the model, will lead to further analysis on the end-forming heating stage, allowing to carry out a characterization of the process in terms of heating time. Considering the high pipe production rate of companies which work in this industrial field and the high electric power installed (around $16 \mathrm{~kW}$ per oven), the creation of such a model can lead to more efficient process able to fulfil the production requirements with the least possible energy consumption.

\section{Outline of the end-forming process}

End-forming indicates a manufacturing operation through which a tube is deformed at one end, so as to allow the connection of several units to form long pipelines, used for the transport of fluids such as waste-water. First the pipe is placed inside an oven to undergo the heating stage, which softens the material thus allowing the deformation phase to be carried out by means of pressurized air or mechanical devices; the end of the process is a cooling step which freezes the pipe in its final shape. The detailed analysis of the end-forming heating phase is the focus of this research work.

\section{The oven}

During manufacturing, heating usually takes place in ovens equipped with infrared lamps arranged radially outside and inside the pipe, as shown in Fig. 1. The emitters are usually coupled quartz tubes of elliptical cross-section, which allow to maximize the power output. These devices are chosen according to the type of polymer to be heated, selecting emission wavelength to match the absorption characteristics of pipe material; therefore, they emit most of their radiative power in narrow bands. Considering short wave quartz emitters, these have little more than $91 \%$ of their radiative power limited to wavelengths below $4 \mu \mathrm{m}$ with about $62 \%$ below $2 \mu \mathrm{m}$ and about $29 \%$ within the $2-4 \mu \mathrm{m}$ band; therefore, the emissivity of short-wave (SW) lamps can be considered uniform for all practical purposes [13]. In addition, the assumption of uniform planar source can be justified by the planar emitting surface of the luminary. Moreover, as shown in Fig. 1, the oven is usually provided with air vents which allow the air propelled by a fan to flow through the heating chamber; thus, in combination with a proper surface temperature control, the operator can adjust the air flow to prevent pipe burnout during the process. In this work, an oven with eight short-wave radially-arranged lamps has been considered. Two additional lamps located inside the tube and angularly displaced by $180^{\circ}$ provide further thermal power when processing tubes with large diameters and thicknesses, as shown in Fig. 1. A rotary motion is imposed on the tube with a certain angular velocity, which determines the angular distribution of thermal radiation incident at the pipe outer surface. Infrared lamps with a rated power $P_{\mathrm{r}}=1000 \mathrm{~W}$, a total length $L_{\mathrm{t}}=430 \mathrm{~mm}$ and a heating length $L_{\mathrm{h}}=300 \mathrm{~mm}$ have been considered.

\section{Material}

The end-forming process can be applied to pipes of different materials; in this study, the thermal analysis focused on polyvinyl chloride (PVC) tubes. Thermophysical characteristics considered for PVC are: density $\rho=1.44 \mathrm{~g} \mathrm{~cm}^{-3}$, thermal conductivity $k=0.18 \mathrm{~W} \mathrm{~m}^{-1} \mathrm{~K}^{-1}$, specific thermal capacity $c=1005 \mathrm{~J} \mathrm{~kg}^{-1} \mathrm{~K}^{-1}$, softening temperature $T_{s}=80^{\circ} \mathrm{C}$, surface emissivity $\varepsilon=0.93$ and absorbtion coefficient for infrared radiation $K_{\mathrm{a}}=147 \mathrm{~m}^{-1}$. PVC has a semitransparent behaviour in the emission band of SW lamps, making the absorption process of the radiation a volumetric 
Fig. 1 Oven with 8 short-wave infrared lamps and 2 additional lamps positioned internally for larger-diameter tubes

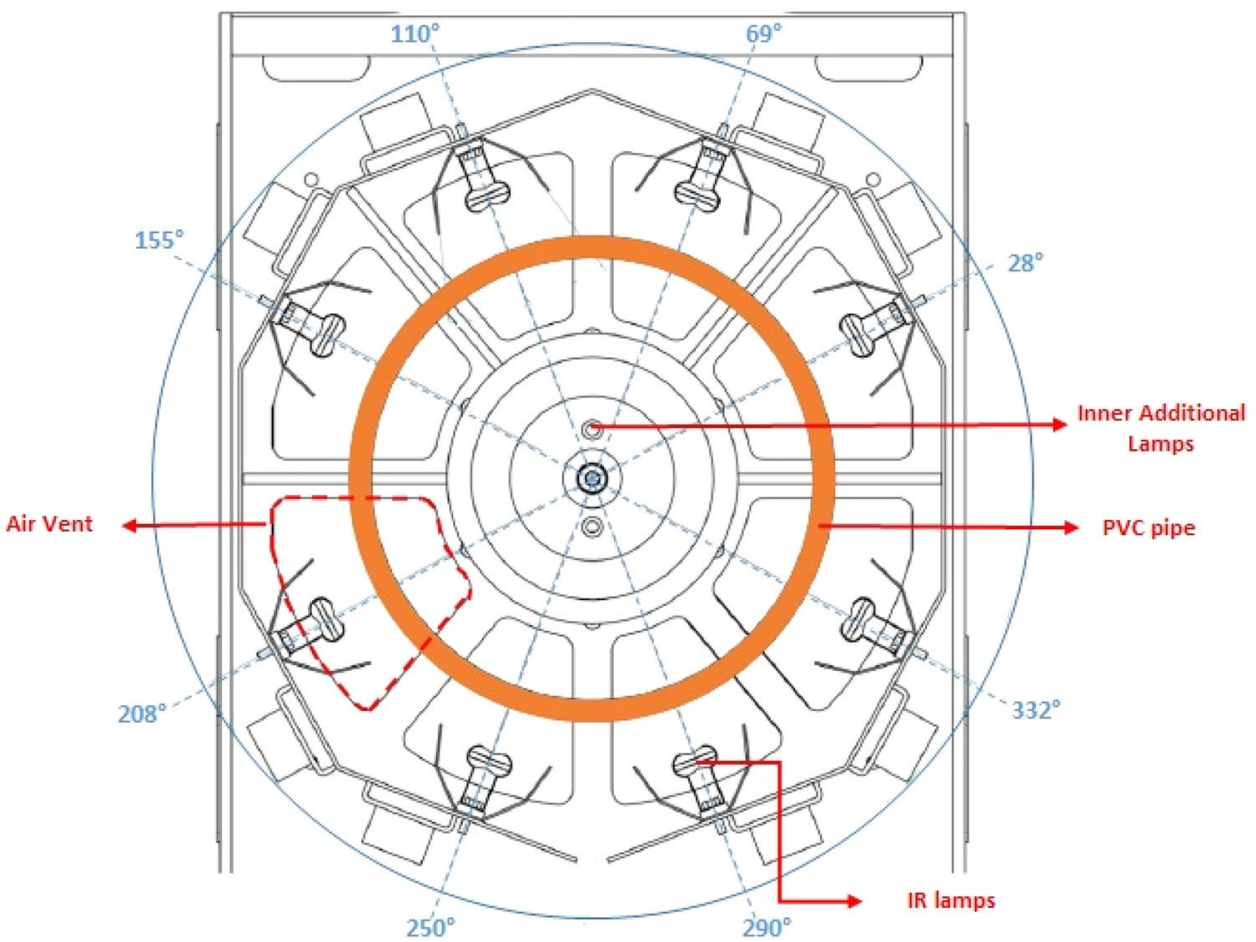

phenomenon which can be described using Lambert's law, as discussed in the next section. $K_{\mathrm{a}}$ represents a very important material property because it directly affects the quantity of energy absorbed by the material when considering a certain kind of emitter. The value chosen for $K_{\mathrm{a}}$ has been established on the basis of information given by manufacturers working in this field. During the actual heating process, attention must be paid to the maximum temperature reached in order to avoid thermal degradation and decomposition of the material; for PVC, decomposition generally manifests as a dehydrochlorination process, which consists of hydrogen chloride production and, for commercial PVC, often starts at temperatures in the range 520-590 K, [14]. Moreover, when reaching a temperature level of about $450 \mathrm{~K}$, burns and surface whitening phenomena begin to appear, making the product unacceptable.

\section{Model}

In order to analyze the effect of tube angular velocity on the temperature distribution within the pipe wall, two different models have been devised. One simulates the uniform radiative heating of a static polymeric tube considering the semitransparent behavior of PVC only, the other the radiative heating of a rotating pipe considering the actual angular distribution of the radiation over the tube. The former model can be seen as an asymptotic form of the latter (e.g. $\omega \longrightarrow+\infty)$. For both models, the input data are the material properties (density $\rho$, thermal conductivity $k$, thermal capacity $c_{p}$, absorption coefficient in the infrared wavelengths $K_{\mathrm{a}}$ and surface emissivity $\varepsilon$ ), pipe geometry (inner radius $r_{\mathrm{i}}$ and outer radius $r_{\mathrm{e}}$ ) and oven configuration (lamp nominal power and heating length, number of external and inner auxiliary lamps, lamps' radial positions $R_{\mathrm{L}}$, lamps' angular positions $\alpha_{\mathrm{L}}$, lamps' length $L$, see Figs. 1, 2). While for the model with uniform radiation it is enough to calculate a global view factor between lamps and pipe on the basis of correlation available in literature [15], in order to simulate the radiative heat exchange between a rotating pipe and the lamps, the angular distribution of the view factors as a function of tube outer radius and oven configuration must be determined, as will be explained in the next section.

\section{View Factor Calculation}

The procedure for the calculation of local view factors for a given pipe geometry and oven configuration has been extensively reported in [12], where the local view factor along the outer circumference of the pipe has been calculated on the basis of the theoretical fundamentals available in [15] for radiative heat exchange between two infinitely long surfaces. Figure 2 shows a sketch of the geometric configuration studied. The eight short-wave lamps in the oven for the end-forming process considered have $L=23 \mathrm{~mm}$ and are positioned at a radial coordinate $R_{L}=143 \mathrm{~mm}$, with a resulting ratio $L / R_{\mathrm{L}}$ equal to 0.16 . As regards the lamps' angular positions $\alpha_{\mathrm{L}}$, a first analysis has been carried out considering 


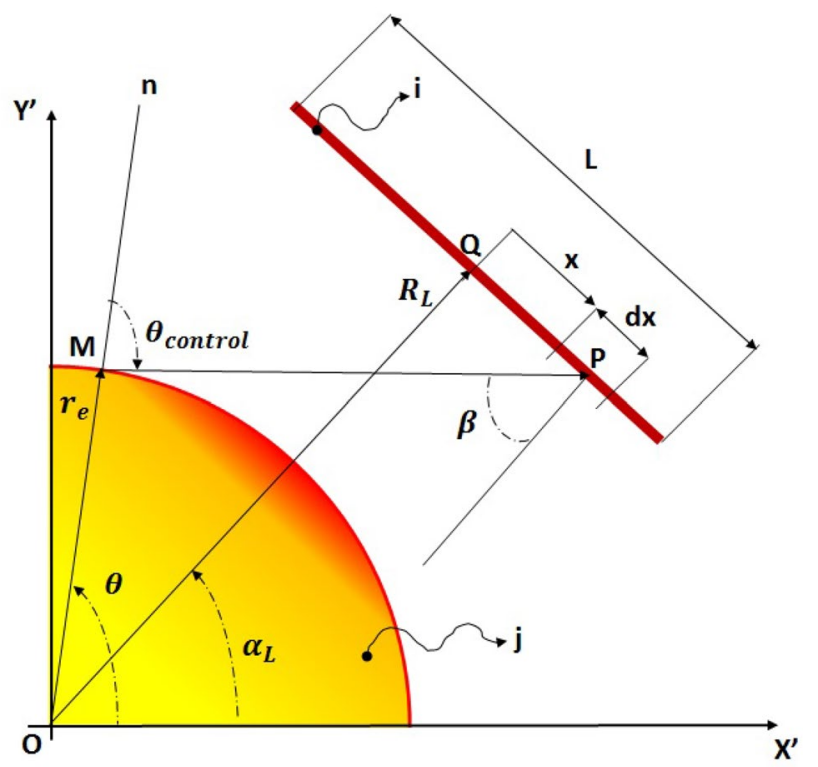

Fig. 2 Geometry for the calculation of local view factors along the external circumference of cylinders

their current placement within a real oven (Fig. 1), in which $\alpha_{\mathrm{L}}=28^{\circ}, 69^{\circ}, 110^{\circ}, 155^{\circ}, 208^{\circ}, 250^{\circ}, 290^{\circ}, 332^{\circ}$.

In the following, the main steps described in [12] will be reported.

The portion of energy leaving an infinitesimal element on the external surface of the cylinder $j$ at an angular position $\vartheta$ which reaches an infinitesimal element of extension $\mathrm{d} x$ on a generic lamp $i$ has been calculated as:

$\mathrm{d} F_{d j \rightarrow d i}=\frac{1}{2} \cdot \mathrm{d}\left(\sin \left(\vartheta_{\text {control }}\right)\right)$

Referring to Fig. 2, $\vartheta_{\text {control }}$ represents the angle between the normal to the outer surface of the pipe on a generic point $M$ and the vector $\overrightarrow{M P}$, where $P$ is a generic point on the surface of the lamp.

After introducing the non-dimensional quantities $\bar{x}=x / R_{\mathrm{L}}$ and $\bar{a}=r_{\mathrm{e}} / R_{\mathrm{L}}$, Eq. (1) takes the following form:

$\mathrm{d} F_{d j \rightarrow d i}=\frac{1}{4}(1+g) \frac{\left[\cos \left(\vartheta-\alpha_{\mathrm{L}}\right)-\bar{a}-\bar{x}\left(1-\bar{a} \cos \left(\vartheta-\alpha_{\mathrm{L}}\right)\right) \sin \left(\vartheta-\alpha_{\mathrm{L}}\right)\right]}{\left[1+\bar{x}^{2}+\bar{a}^{2}+2 \bar{a}\left(\bar{x} \sin \left(\vartheta-\alpha_{\mathrm{L}}\right)-\cos \left(\vartheta-\alpha_{\mathrm{L}}\right)\right]^{\frac{3}{2}}\right.} \mathrm{d} \bar{x}$

In Eq. (2) $g$ represents a control function defined as $g=\operatorname{sign}(\overrightarrow{O M} \cdot \overrightarrow{M P})$, which accounts for the portion of cylinder surface in the shadow zone of the i-th lamp.

Numerically integrating Eq. (2) between $\bar{x}_{1}=-L / 2 R_{\mathrm{L}}$ and $\bar{x}_{2}=+L / 2 R_{\mathrm{L}}$ leads to $F_{d j \rightarrow i}$ :

$F_{d j \rightarrow i}=\int_{\bar{x}_{1}}^{\bar{x}_{2}} \mathrm{~d} F_{d j \rightarrow d i}$
The quantity obtained in Eq. (3) represents the fraction of energy leaving an infinitesimal element on the external surface of the cylinder $j$ at an angular position $\vartheta$ which reaches the $i$ th lamp of finite extension $L$.

Reciprocity law has been subsequently used, as shown in Eq. (4), allowing to calculate the fraction of energy $F_{i \rightarrow d j}$ leaving the $i$ th lamp of finite extension $L$ which reaches an infinitesimal element of extension $r_{\mathrm{e}} \cdot d \vartheta$ on the outer surface of the cylinder $j$ at an angular position $\vartheta$.

$F_{i \rightarrow d j}=F_{d j \rightarrow i} \frac{r_{\mathrm{e}} \cdot \mathrm{d} \vartheta}{L}$

To have a distribution of the view factors on the outer side of the cylinder, the outer circumference must be discretized into $N$ angular arcs, each of angular extension $\Delta \vartheta=2 \pi / N$. The fraction of energy leaving the $i$ th lamp of finite extension $L$ which reaches an angular arc of finite extension $r_{\mathrm{e}} \cdot \Delta \vartheta$ can be calculated as:

$F_{i \rightarrow j}=\frac{r_{\mathrm{e}}}{L} \int_{\vartheta-\frac{\Delta \vartheta}{2}}^{\vartheta+\frac{\Delta \vartheta}{2}} \int_{\bar{x}_{1}}^{\bar{x}_{2}} \mathrm{~d} F_{d j \rightarrow d i} \mathrm{~d} \vartheta$

To this aim, the outer circumference of the cylinder has been discretized into $N=1000$ arcs of equal angular extension $\Delta \vartheta$. Equation (5) has been numerically integrated by means of the MATLAB ${ }^{\circledR}$ function integral2, $[16,17]$. Extending the procedure described before to each arc, the view factor distribution on the outer circumference of a cylinder for a given layout of the lamps was calculated. Figure 3 represents the distribution of the local view factors for three different external radii $r_{\mathrm{e}}$ in the case of a single lamp, Fig. 3a, and in the case of eight lamps angularly displaced as in the real oven, Fig. 3b, when considering a ratio $L / R_{\mathrm{L}}$ equal to 0.16 . The validation of the numerical procedure to calculate the local view factor profile has been carried out comparing the global view factor, calculated as the sum of all local view factors, with the value obtained by means of the corresponding expression available in the literature [15]. The numerical approach developed by the authors gave the same results as those obtained using the analytical expression, thus validating our mathematical model.

\section{Lambert's law for the semitransparent behaviour of PVC}

Considering the semitransparent behaviour of PVC within the emission band of SW lamps, thermal irradiation $I_{\mathrm{r}}$ transferred at a given radius $r$ can be expressed as:

$I_{\mathrm{r}}=I_{r_{\mathrm{e}}} \cdot \exp \left(-K_{\mathrm{a}} \cdot\left(r_{\mathrm{e}}-r\right)\right)$

Equation 6 describes Lambert's law and relates the thermal irradiation $I_{\mathrm{r}}$ to the incident radiative flux $I_{\mathrm{re}}$ at the outer radius $r_{\mathrm{e}}$, the coefficient of absorption $K_{\mathrm{a}}$, the outer radius $r_{\mathrm{e}}$ 


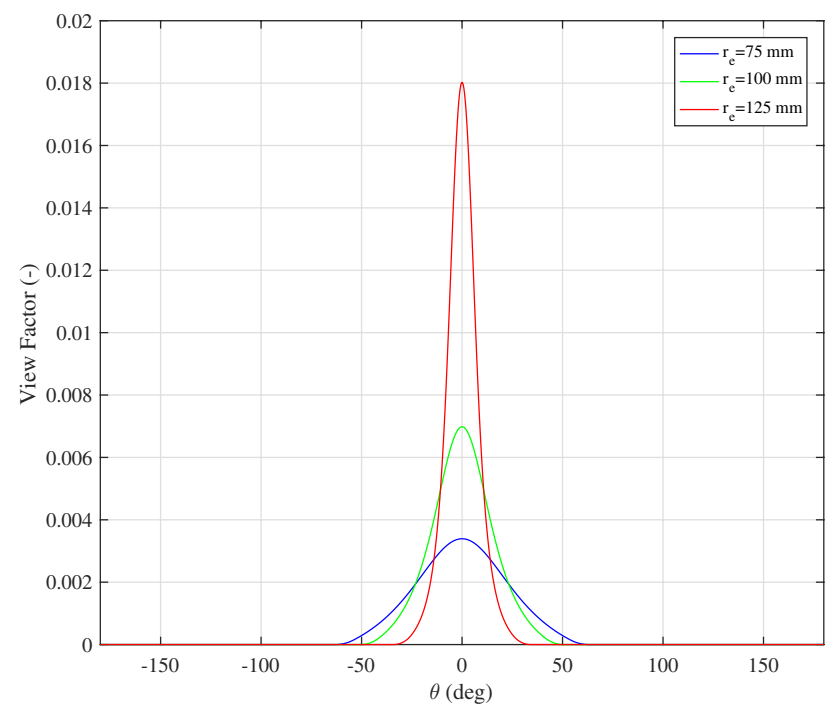

(a)

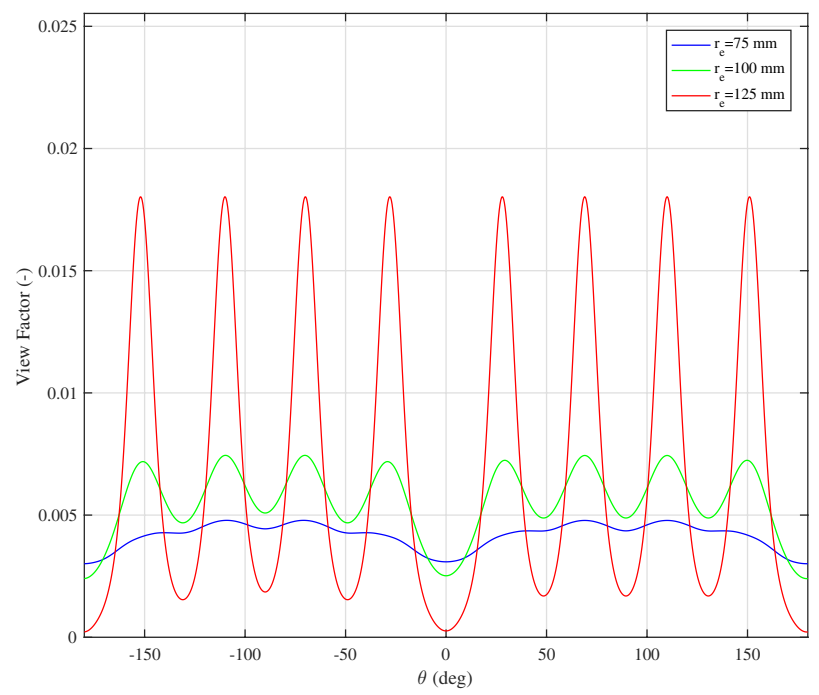

(b)

Fig. 3 Local view factors. a Single lamp configuration. b Eight lamps configuration

and the generic radius $r$. As previously stated, the radiative heating of semitransparent media is a volumetric phenomenon and can therefore be simulated as a heat source term. Based on the discussion in [15], the heat source term due to external lamps radiation in cylindrical coordinates can be expressed as:

$q_{\mathrm{g}}^{\prime \prime \prime}=\left(\frac{1}{r}+K_{\mathrm{a}}\right) \cdot I_{r_{\mathrm{e}}} \cdot \exp \left(-K_{\mathrm{a}} \cdot\left(r_{\mathrm{e}}-r\right)\right)$

As discussed in "The oven" section, for tubes with large diameters and thicknesses additional lamps positioned on the inner side of the pipes are used. When present, the heat source term due to internal lamps radiation in cylindrical coordinates can be calculated from:

$q_{\mathrm{g} \text { Internal Lamps }}^{\prime \prime \prime}=\left(K_{\mathrm{a}}-\frac{1}{r}\right) \cdot I_{\mathrm{ri}} \cdot \exp \left(-K_{\mathrm{a}} \cdot\left(r-r_{\mathrm{i}}\right)\right)$

$I_{\mathrm{ri}}$ indicates the radiative heat flux incident at pipe inner surface and $r_{\mathrm{i}}$ the inner radius.

\section{Finite element model}

After an expression for the calculation of local view factors along the outer circumference of the cylinder was obtained, a finite element model accounting for cylinder rotation via a time-dependent boundary condition applied to a stationary domain was set-up in $\mathrm{COMSOL}^{\circledR}$. In the uniform radiation model, the heat source term depends on the radial coordinate only, while in the rotating cylinder model it depends both on the radial and angular coordinates of a cylindrical reference system and is considered as time-varying with a time period equal to $2 \pi / \omega$, where $\omega$ is the angular velocity of the PVC tube. The local heat source intensity at any given time instant was computed by the solver recalling the MATLAB ${ }^{\circledR}$ function written for the calculation of the local view factors. As reported in [12], the influence of axial thermal conduction on the temperature distribution within the tube wall was investigated developing a 3D model: in an opposite way to other thermal problems [18-22], simulations demonstrated how conjugate effects affect little the temperature field in the heated zone owing to the high conductive thermal resistance of the PVC tube. The analysis on the effects of tube angular velocity on the temperature distribution has therefore been carried out over 2D domains. Equation (9) describes the thermal energy transport in a solid medium under transient conditions, considering a generic $2 \mathrm{D}$ domain in cylindrical coordinates, with $r$ the radial coordinate, $z$ the axial coordinate and $\vartheta$ the angular coordinate respectively.

$\frac{1}{\alpha} \frac{\partial T}{\partial \tau}=\frac{q_{\mathrm{g}}^{\prime \prime \prime}(r, \vartheta, \tau)}{k}+\frac{\partial^{2} T}{\partial r^{2}}+\frac{1}{r} \frac{\partial T}{\partial r}+\frac{1}{r^{2}} \frac{\partial^{2} T}{\partial \vartheta^{2}}$

A comparison with the case of uniform radiative flux was made in terms of the maximum temperature difference along the outer circumference, as well as the circumference defined by the mean radius and the inner circumference. The term corresponding to internal generation $q_{\mathrm{g}}^{\prime \prime \prime}$ due to external lamps radiation can be calculated by means of Eq. 7 and depends on the radial coordinate $r$ only in the model with uniform radiation, while it is a function of $r, \vartheta$ and time, $\tau$, when considering the view factor distribution due to pipe geometry and oven configuration for the calculation of $I_{\mathrm{re}}$. The heat generation due to internal lamps, when present, has 
been considered uniform in $\vartheta$ and expressed by means of Eq. (8).

When $\tau=\tau_{0}=0 \mathrm{~s}$, the temperature at each point in the domain is uniform, $T_{0}=293.15 \mathrm{~K}$. At the inner radius, assuming that no axial air flow was present, a boundary condition of adiabaticity was considered, because of the very low value of the convection heat transfer coefficient obtained by means of the correlation experimentally given in [9] for a rotating horizontal cylinder. At the outer radius, a combined convective-radiative boundary condition was adopted, with an external convective coefficient $h_{\mathrm{e}}$ calculated with the correlation given by Etemad for natural convection on horizontal rotating cylinders [23], neglecting the presence of an axial air flow over the pipe outer surface. In fact, the value of pipe surface emissivity $\varepsilon$ and the convective heat transfer coefficients, which contribute to the definition of the boundary conditions, do not have a significant influence on temperature unevenness within the pipe, as shown in [12]. The temperatures of ambient air, $T_{\text {air }}$, and walls, $T_{\mathrm{w}}$, were both considered at $293.15 \mathrm{~K}$.

\section{Model reliability and sensitivity analysis}

Before starting with simulations aimed at quantifying the temperature displacement with respect to the case of a perfectly uniform radiation as a function of angular velocity and oven configuration, the reliability of the model has been investigated comparing the asymptotic solution of the timedependent 2D finite-element model in the case of uniform heat flux with the analytical solution obtained integrating the heat equation in its one-dimensional and axisymmetric form and in steady-state condition. Neglecting angular variations in temperature field and imposing steady-state conditions, Eq. (9) becomes:

$\frac{\partial^{2} T}{\partial r^{2}}+\frac{1}{r} \frac{\partial T}{\partial r}+\frac{q_{\mathrm{g}}^{\prime \prime \prime}(r, \vartheta, \tau)}{k}=0$

Integrating Eq. (10) twice, considering external SW lamps only and assuming conditions of adiabaticity at the inner radius and a convective boundary condition at the outer radius leads to the steady-state temperature profile within pipe wall:

$$
\begin{aligned}
T(r)= & T_{\text {air }}+I_{\mathrm{re}} \cdot\left(\frac{1}{k K_{\mathrm{a}}}+\frac{1}{h_{\mathrm{e}}}\right)-\frac{I_{\mathrm{re}} r_{\mathrm{i}}}{h_{\mathrm{e}} r_{\mathrm{e}}} \cdot \exp \left(-K_{\mathrm{a}}\left(r_{\mathrm{e}}-r_{\mathrm{i}}\right)\right) \\
& +\frac{I_{\mathrm{re}} r_{\mathrm{i}}}{k} \cdot \exp \left(-K_{\mathrm{a}}\left(r_{\mathrm{e}}-r_{\mathrm{i}}\right)\right) \cdot \ln \left(\frac{r}{r_{\mathrm{e}}}\right)-\frac{I_{\mathrm{re}}}{k K_{\mathrm{a}}} \cdot \exp \left(-K_{\mathrm{a}}\left(r_{\mathrm{e}}-r\right)\right)
\end{aligned}
$$

The asymptotic solution obtained by means of the timedependent 2D finite-element model showed perfect agreement with the analytical steady-state solution described by Eq. (11), giving maximum discrepancies lower than $0.03 \%$ over the whole domain.
Moreover, a grid-sensitivity analysis was conducted, considering quadrilateral Lagrange elements. To control grid refinement, the number of elements on the outer circumference of the cylinder, $N_{\mathrm{El}}$ OC , has been chosen as tunable parameter. To establish the most suitable value of $N_{\mathrm{El} \mathrm{OC}}$, the temperature at the end of a $100 \mathrm{~s}$ simulation on a point placed in the middle of pipe wall $\left(r=r_{\mathrm{m}}\right)$ and at the angular position $\vartheta=0^{\circ}$ was monitored. The grid-sensitivity analysis was conducted on a tube with $D_{e}=250 \mathrm{~mm}$ and thickness th $=13.4 \mathrm{~mm}$ since it represents one of the largest processable pipe in terms of diameter and thickness and it is reasonably more affected by angular and radial thermal gradients than smaller pipes. In addition, three different operating conditions were considered: stationary pipe, rotating pipe with $\omega=1.87 \mathrm{rad} \mathrm{s}^{-1}$ and pipe irradiated by a perfectly uniform radiation. Figure 4 shows the results of the grid-sensitivity analysis; in particular, the temperature of the monitored point in the three operating conditions at the end of the simulation and the total number of elements in the grid, $N_{\mathrm{El} \mathrm{Tot}}$, are reported. The case of rotating pipe represents the most critical one, showing grid independence for $N_{\mathrm{El} \mathrm{OC}} \geq 500$; thus, 500 has been subsequently chosen as the best value for $N_{\mathrm{El} \mathrm{OC}}$, since it was verified that it guaranteed grid independence for smaller pipes too. For a pipe with outer diameter $D_{e}=250 \mathrm{~mm}$ and thickness th $=10.8 \mathrm{~mm}$, which is the largest geometry investigated, a mesh formed by 3996 quadrilateral Lagrange elements and 4496 nodes was subsequently adopted.

The temporal discretization method was the Alpha method and the maximum time-step adopted was $0.1 \mathrm{~s}$, defined after a time-sensitivity analysis. Simulations aimed at quantifying the temperature unevenness within pipe wall had a duration of $100 \mathrm{~s}$.

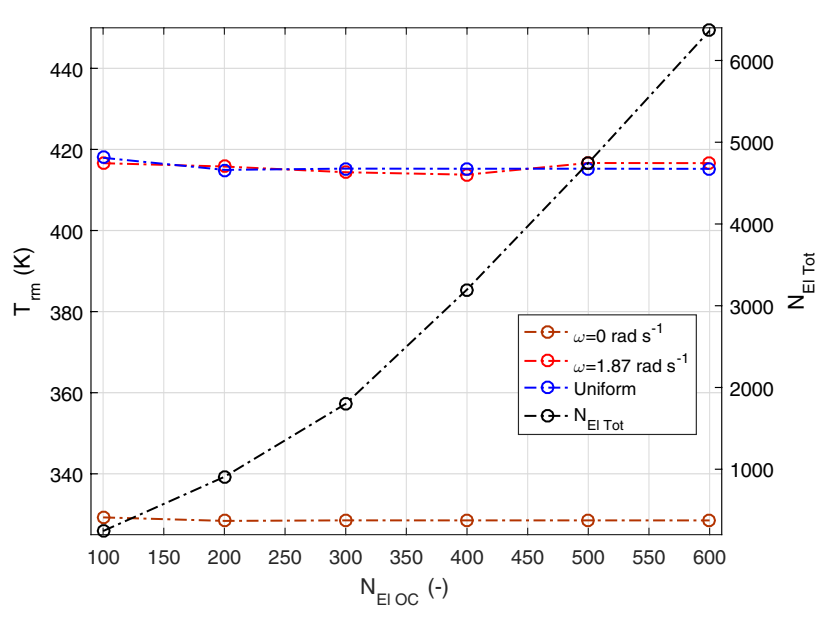

Fig. 4 Grid sensitivity analysis. Temperature on a point placed in the middle of pipe wall $\left(r=r_{\mathrm{m}}\right.$ and $\left.\vartheta=0^{\circ}\right)$ and total number of elements in the grid. $D_{e}=250 \mathrm{~mm}$, th $=13.4 \mathrm{~mm}$ 


\section{Results}

In Fig. 5 the temperature profile along the outer circumference of a stationary PVC pipe in a oven like that shown in Fig. 1 has been compared at different times with that due to uniform radiation. A tube with $D_{e}=250 \mathrm{~mm}$ and th $=10.8 \mathrm{~mm}$ has been investigated, since it represents one of the largest customary tube sizes in end-forming processes. An external convection coefficient $h_{\mathrm{e}}=9 \mathrm{~W} \mathrm{~m}^{-2} \mathrm{~K}^{-1}$ was calculated. Two additional internal SW lamps have been considered, in order to make the heating process quicker and more uniform, as usually happens in practice. The actual temperature on the outer circumference is strongly affected by the view factor distribution: at $\tau=40 \mathrm{~s}$, the maximum temperature exceeds $500 \mathrm{~K}$, closely approaching the limit of dehydrochlorination, with a maximum temperature difference of $120 \mathrm{~K}$ with respect to the uniform radiation case. This result fully justifies the use of handling systems for the rotation of tubes, in order to obtain uniform heating.

Simulations at different angular velocities were carried out for the same PVC tube. Figure 6 shows the temperature profile within pipe wall when considering the maximum angular velocity investigated $\left(\omega=1.87 \mathrm{rad} \mathrm{s}^{-1}\right)$. It can be clearly seen that the maximum temperature shifts inwards from the outer wall, because of the semitransparent behavior of PVC with respect to short-wave radiation. As time increases, the maximum temperature moves toward the inner radius, because of the increase in convective heat exchange at the outer surface. The flat trend of curves at the inner radius is a consequence of the assumption of adiabaticity, because of the small influence of the drag effect caused by the tube rotation on the air motion, when axial air flow is absent.

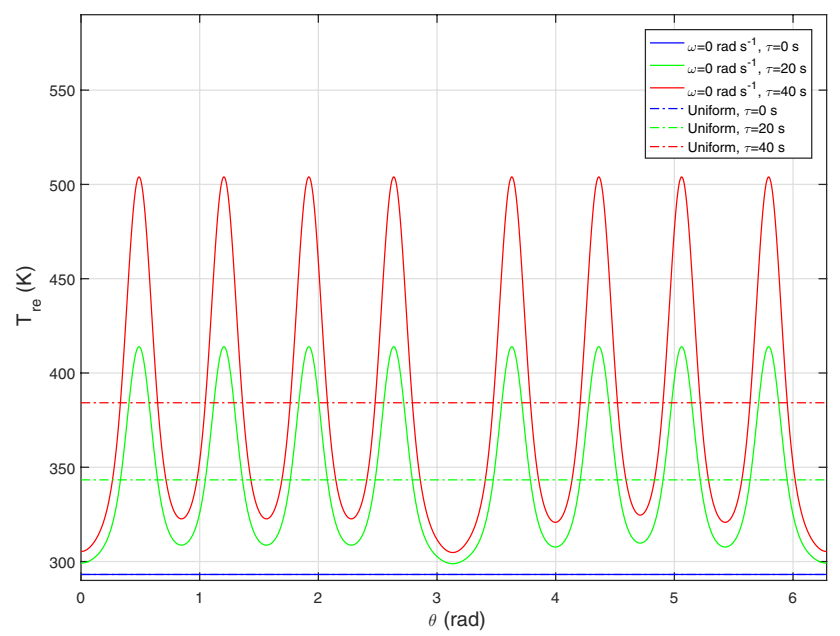

Fig. 5 Temperature profile along the outer circumference of a stationary cylinder. $D_{e}=250 \mathrm{~mm}$, th $=10.8 \mathrm{~mm}$

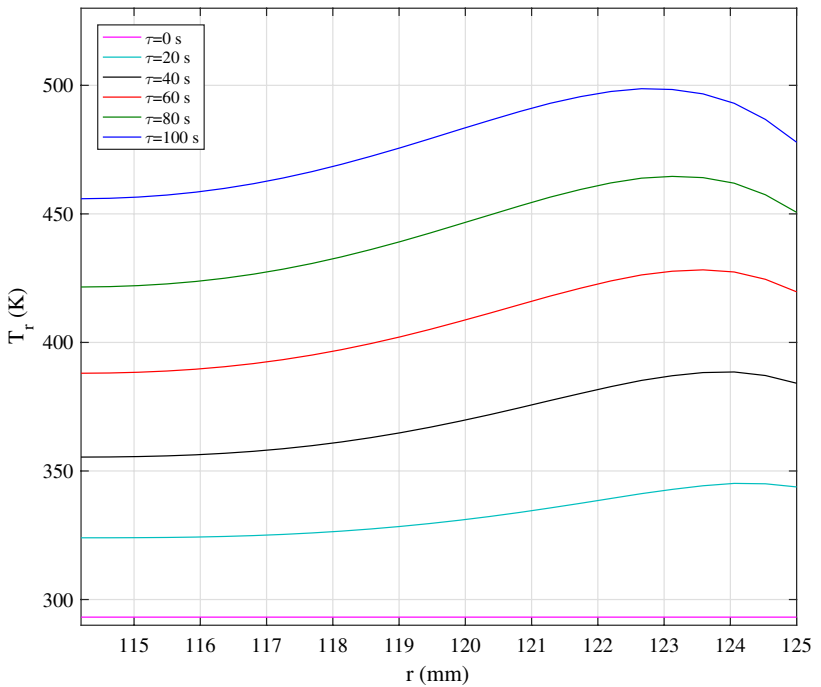

Fig. 6 Temperature distribution within pipe wall. $D_{e}=250 \mathrm{~mm}$, th $=10.8 \mathrm{~mm}, \omega=1.87 \mathrm{rad} \mathrm{s}^{-1}$

Figure 7 depicts the maximum temperature displacement along the outer circumference, $\Delta T_{r_{\mathrm{e}}}$ : as expected, this quantity decreases with increasing angular velocity. Moreover, curves show a nonlinear dependence of $\Delta T_{r_{\mathrm{e}}}$ on the angular velocity: the reduction of $\Delta T_{r_{\mathrm{e}}}$ with increasing angular velocity is more pronounced for low values of $\omega$. This implies the existence of a limiting angular velocity, over which a further increase does not bring substantial reductions in the maximum temperature displacement.

Figure $8 \mathrm{a}, \mathrm{b}$ show the same quantity along the circumference defined by the mean radius $\left(\Delta T_{r_{\mathrm{m}}}\right)$ and along the inner circumference $\left(\Delta T_{r_{\mathrm{i}}}\right)$. A reduction in the maximum

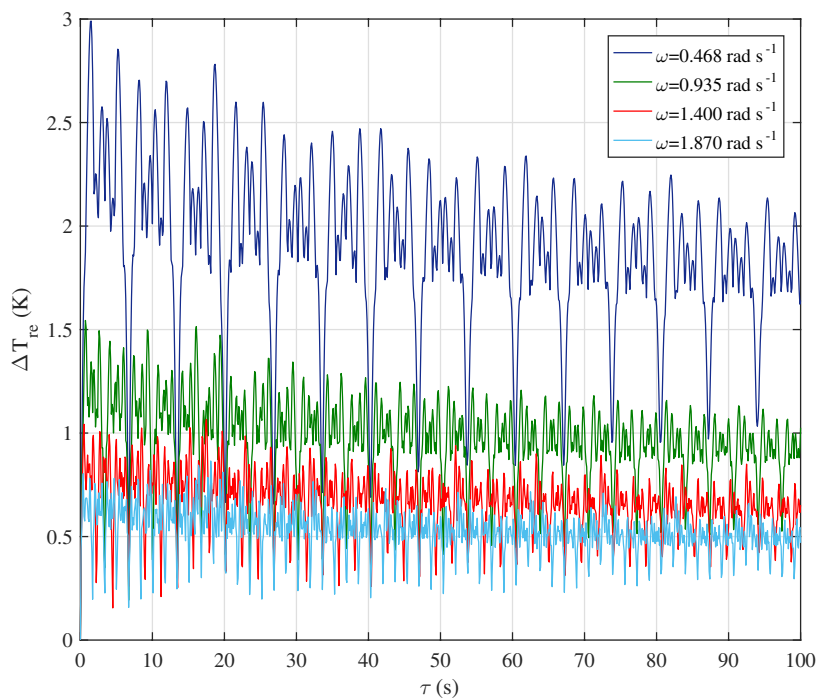

Fig. 7 Maximum temperature displacement along the outer circumference. $D_{e}=250 \mathrm{~mm}$, th $=10.8 \mathrm{~mm}$ 


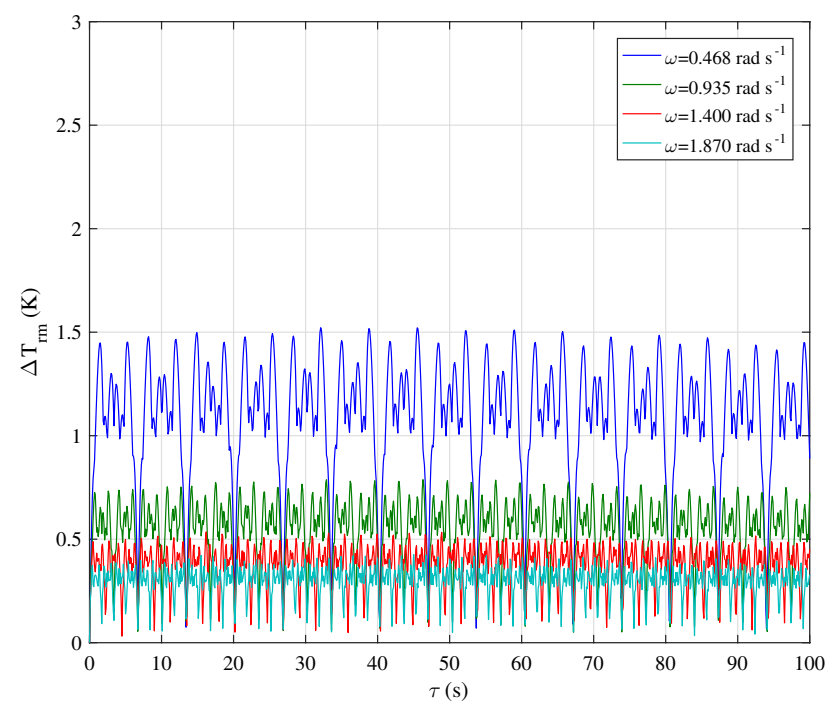

(a)

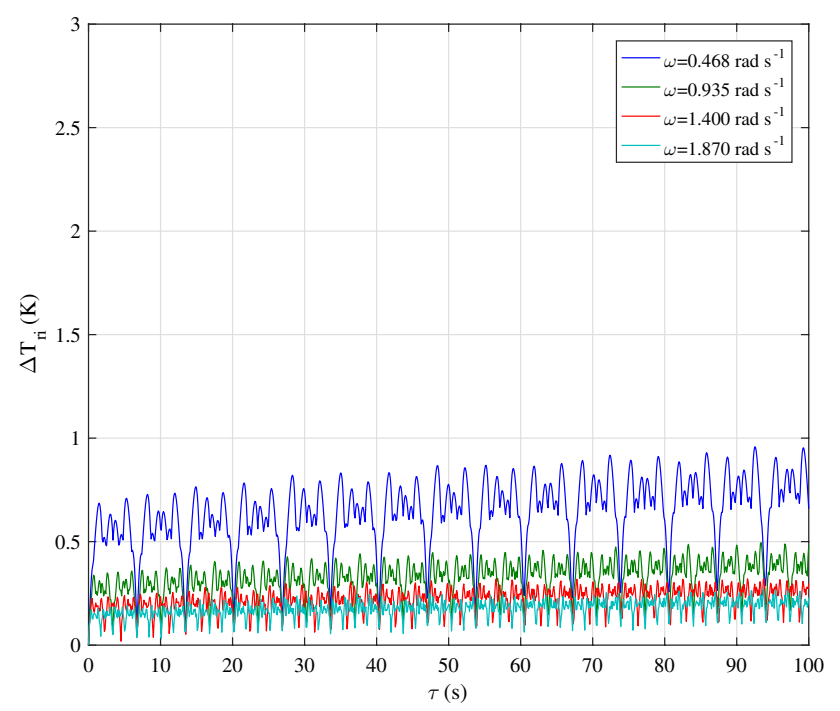

(b)

Fig. 8 Maximum temperature displacement along the circumference defined by the mean radius (a) and along the inner circumference (b). $D_{e}=250 \mathrm{~mm}$, th $=10.8 \mathrm{~mm}$

temperature displacement when moving to lower radii appears for each angular velocity investigated. This behavior is more pronounced at low angular velocities and it is due to the low thermal diffusivity of the material which dampens the amplitude of thermal perturbations and imposes a time delay to it, which causes an increase in the temperature displacement at the inner radius when time progresses.

A set of simulations with the same boundary conditions but without internal lamps was run for a tube with $D_{e}=125 \mathrm{~mm}$ and th $=7.4 \mathrm{~mm}$, which represents a mediumsized tube. Figure 9 shows the trend of $\Delta T_{r_{\mathrm{e}}}$ for different values of angular velocities. Comparing the curves in Fig. 9 with those in Fig. 7 and considering the same values of

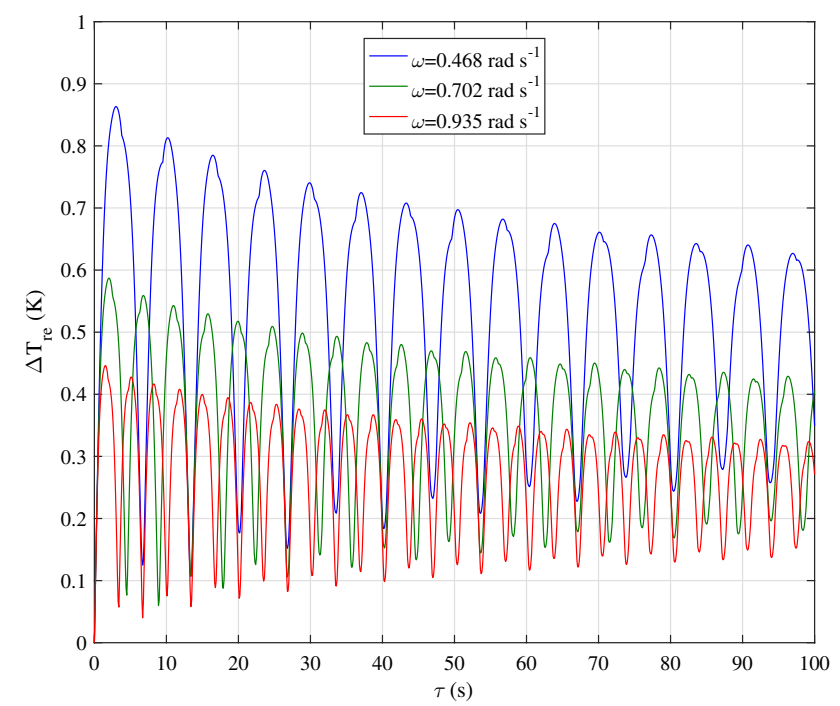

Fig. 9 Maximum temperature displacement along the outer circumference. $D_{e}=125 \mathrm{~mm}$ and th $=7.4 \mathrm{~mm}$ thick

angular velocity, a strong reduction in $\Delta T_{r_{\mathrm{e}}}$ emerges with decreasing external diameter; this can be justified considering the local view factor distribution, which becomes less affected by the angular position as the diameter of the pipe decreases; see Fig. 3b. This means that for pipes of smaller dimensions, the limiting value of angular velocity takes lower values.

A critical angular velocity can be defined as the minimum value of rotational speed which allows to maintain the maximum temperature displacement below a certain threshold. For a given oven configuration, the model can be used to determine this critical value for different tube geometries, after a maximum allowable temperature displacement $\left(\Delta T_{\max }\right)$ along the outer circumference with respect to a perfectly uniform heating process has been established. To this aim, for each tube geometry and angular velocity investigated, $\Delta T_{\max }$ must be compared to $\Delta T_{r_{\mathrm{e}}}$, whose value is time-dependent, as clearly demonstrated in Figs. 7 and 9, because of the combined effects of conduction and convection which become more pronounced at high temperatures, favoring reduction in peak values of $\Delta T_{r_{\mathrm{e}}}$. In order to establish a significant instantaneous value of $\Delta T_{r_{\mathrm{e}}}$ for the comparison with $\Delta T_{\max }$, a time interval $\Delta \tau=\left[\tau_{0}-p, \tau_{0}+p\right]$ has been considered, where $p$ is lamps facing period, defined as $p=2 \pi /\left(\omega \cdot N_{\text {lamp }}\right)$, where $N_{\text {lamp }}$ is the number of external lamps in the oven, and $\tau_{0}$ is the instant of time in which the mean temperature along the outer circumference reaches the value $T_{\text {off }}=433.15 \mathrm{~K}$, which represents a credible value of the switch-off temperature of lamps, when these are controlled by a traditional pyrometer. The comparison value has consequently been calculated as $\Delta T_{\text {eval }}=\max \{\Delta T(\tau)\}$, with $\tau \in \Delta \tau$. The value of $\Delta T_{\max }$ can be established arbitrarily in 
the process design stage: in this work, a maximum temperature displacement $\Delta T_{\max }=1 \mathrm{~K}$ has been considered. The analysis on critical angular velocity has been carried out on three different pipe geometries and considering two different oven configurations in order to investigate the effects of the reciprocal angular positions of the lamps on critical velocity values. As to the latter aspect, the oven with external lamps positioned as described in "View factor calculation" section has been compared to one with eight equally-displaced SW lamps. Figure 10a-c show the trends of $\Delta T_{\text {eval }}$ for three pipe geometries and for the two configurations of lamps considered, comparing it to $\Delta T_{\max }$. It is clear how, for a given geometry and oven configuration, the value of $\Delta T_{\text {eval }}$ decreases when increasing angular velocity and tends to flatten. The intersection between the curves relative to $\Delta T_{\text {eval }}$ and $\Delta T_{\max }$ identifies the value of critical angular velocity, over which a further increase in angular velocity does not lead to significant improvements in the uniformity of the temperature distribution. It is clear how higher radii necessitate higher angular velocities to obtain a maximum temperature displacement of $1 \mathrm{~K}$ with respect to the case of uniform radiation. Moreover, fixing pipe geometry, a substantial reduction in the values of $\Delta T_{\text {eval }}$ and consequently in critical angular velocity is obtained when moving to a configuration with eight lamps displaced at uniform angular intervals. This tendency is stronger for pipes of smaller size.

The larger reduction in critical angular velocity highlighted for smaller pipes in the case of lamps equally-displaced can be justified observing the heat flux profile on the pipes' outer surface for the three geometries investigated and for the oven configurations studied, shown in Fig. 11a-c. In fact, with reference to Fig. 2, for a given lamps radial position $R_{\mathrm{L}}$ and extension $L$, it is clear how pipes with low external diameter are more sensitive to the angular position of the lamps, because of the overlap of the heat fluxes generated by every single lamp, which increases the deviation from the uniform radiation.

\section{Conclusions}

A 2D finite-element model for the transient analysis of the radiative heat exchange between a rotating polymeric cylindrical pipe and the SW infrared lamps of an oven for endforming has been devised in order to investigate the influence of cylinder angular velocity and oven configuration on the temperature distribution within the tube. Results obtained for different rotational velocities were compared with the case of a uniformly irradiated tube. A MATLAB ${ }^{\circledR}$ function for the calculation of local view factor distribution given pipe's outer radius and oven configuration has been developed. The code was validated comparing the global view factor calculated as the sum of all local view factors with the

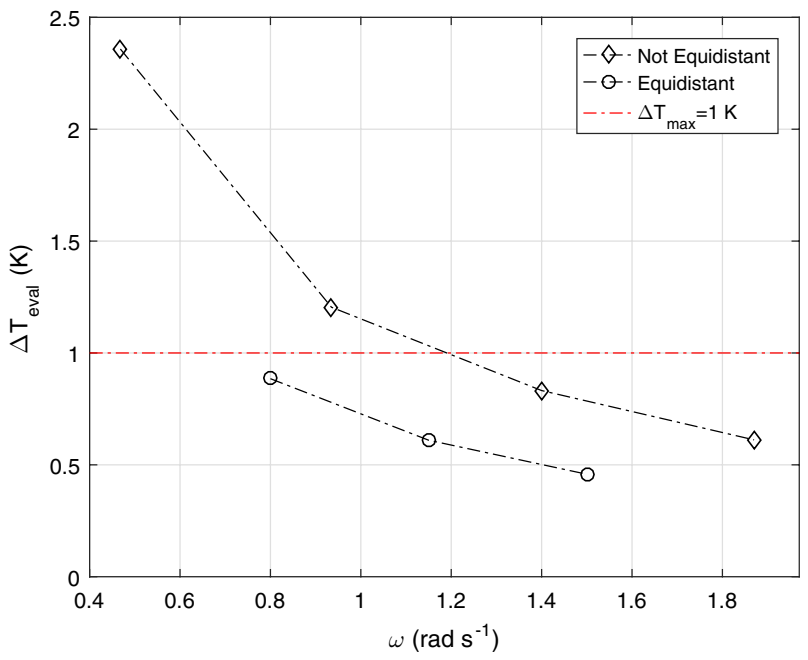

(a)

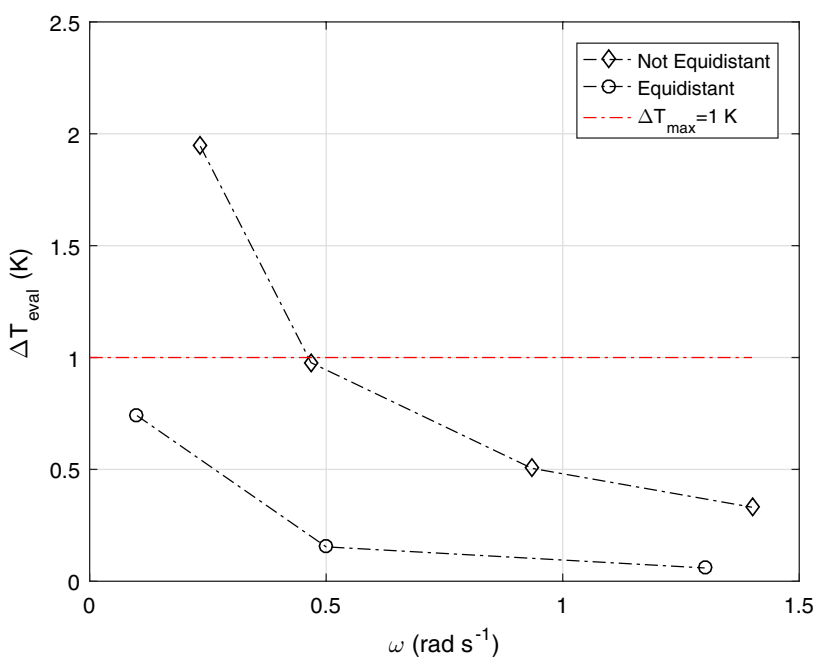

(b)

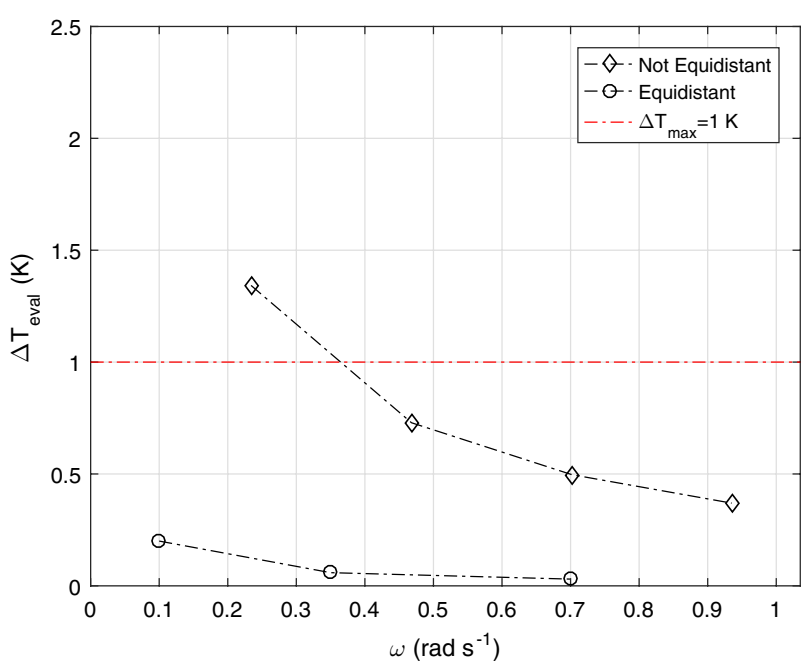

(c)

Fig. 10 Effect of the lamps' angular positions on critical velocity. a Big-size pipe: $D_{e}=250 \mathrm{~mm}$ th $=10.8 \mathrm{~mm}$. b Medium-size pipe: $D_{e}=180 \mathrm{~mm}$ th $=10.7 \mathrm{~mm}$. $\mathbf{c}$ Small-size pipe: $D_{e}=125 \mathrm{~mm}$ th $=7.4 \mathrm{~mm}$ 
Fig. 11 Effect of the lamps' angular positions on heat flux distribution along the outer circumference of pipes. a External diameter of $250 \mathrm{~mm}$ and thickness of $10.8 \mathrm{~mm}$. b External diameter of $180 \mathrm{~mm}$ and thickness of $10.7 \mathrm{~mm}$. c External diameter of $125 \mathrm{~mm}$ and thickness of $7.4 \mathrm{~mm}$

value obtained by means of the corresponding correlation given in [15]. Comparison between the temperature distributions on the outer circumference of a static tube obtained in the cases of uniform and actual radiation distribution justifies the use of rotation devices, in order to avoid high temperature peaks which could lead to the thermal failure of the material. Simulations carried out on different pipe geometries at varying values of angular velocity demonstrated how increasing angular speed decreases the maximum temperature displacement with respect to the case of a uniformly irradiated tube. Results showed a nonlinear dependence between maximum temperature displacement and angular velocity, thus demonstrating the existence of a limiting value over which a further increase in angular velocity brings little reduction in maximum temperature difference. The same analysis also highlighted how the low thermal diffusivity of the material dampens the maximum temperature difference when moving from the outer to the inner side of the pipe. For a fixed value of the angular velocity, lower values of maximum temperature difference were obtained for smaller tubes, because of the more uniform distribution of the local view factors with respect to the angular coordinate. Moreover, as highlighted in [12], parameters which contribute to the definition of boundary conditions, such as convective heat transfer coefficients and PVC surface emissivity, do not influence temperature unevenness significantly over the pipe wall, since they just affect the mean temperature reached at a certain radial position at a given time without varying the maximum temperature displacement with respect to the case of a perfectly uniform radiation. The model can be used as a tool to characterize the end-forming process of polymeric tubes in terms of critical angular velocity, for different materials and oven configurations. Simulations showed that, for a given oven configuration, higher critical velocities are obtained for large-sized pipes, because of the much more uneven distribution of the heat flux on the outer circumference. Also, comparisons between two different oven configurations showed how relative angular positions of lamps could lead to significant modification of the values of critical angular velocity, all the more for smaller pipes.

To the best knowledge of the authors, this is the first numerical model able to characterize the heating stage of the end-forming process in terms of critical angular velocity; an experimental campaign is planned to validate the numerical predictions and fine-tune the model. Finally, it should be mentioned that the addition of surface temperature control in the model can allow the design of optimal heating cycles

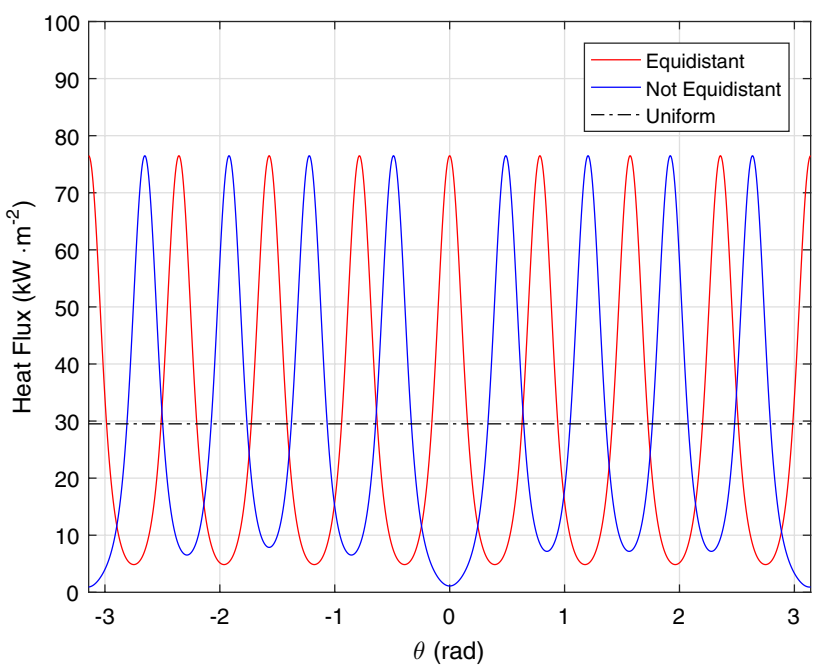

(a)

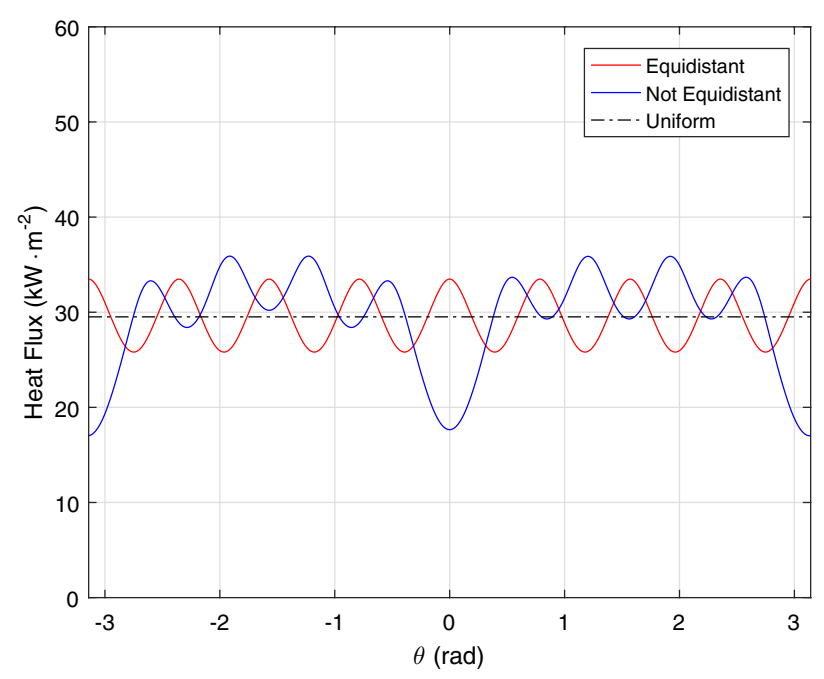

(b)

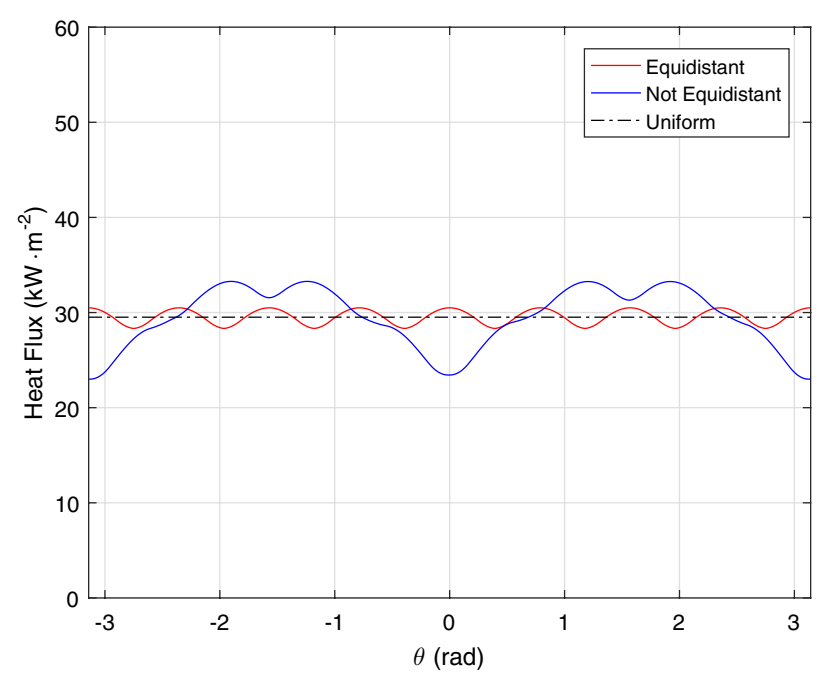

(c) 
to achieve the desired pipe temperature in the most efficient way, meeting production requirements whilst saving energy at the same time.

Open Access This article is distributed under the terms of the Creative Commons Attribution 4.0 International License (http://creativecomm ons.org/licenses/by/4.0/), which permits unrestricted use, distribution, and reproduction in any medium, provided you give appropriate credit to the original author(s) and the source, provide a link to the Creative Commons license, and indicate if changes were made.

\section{References}

1. Bush, S.: Scale, order and complexity in polymer processing. Proc. Inst. Mech. Eng. Part E J. Process. Mech. Eng. 214, 217 (2000)

2. Throne, J.L.: Technology of Thermoforming. Hanser, Munich (1996)

3. Luo, Y., Chevalier, L., Utheza, F., Nicolas, X.: Simplified modelling of the infrared heating involving the air convection effect before the injection stretch blowing moulding of PET preform. Key Eng. Mater. 611-612, 844 (2014)

4. Cosson, B., Schmidt, F., Maoult, Y.L., Bordival, M.: Infrared heating stage simulation of semi-transparent media (PET) using ray tracing method. Int. J. Mater. Form. 4(1), 1 (2011)

5. Sikora, R.: The effect of heating of PVC pipes on selected mechanical properties of pipe bells. Polimery 43, 384 (1998)

6. Fénot, M., Bertin, Y., Dorignac, E., Lalizel, G.: A review of heat transfer between concentric rotating cylinders with or without axial flow. Int. J. Therm. Sci. 50, 1138 (2011)

7. Costa, V., Raimundo, A.: Steady mixed convection in a differentially heated square enclosure with an active rotating circular cylinder. Int. J. Heat Mass Transf. 53, 1208 (2010)

8. Ma, H., Ding, Z., Cao, Y., Lv, X., Lu, W., Shen, X., Yin, L.: Characteristics of the heat transfer from a horizontal rotating cylinder surface. Exp. Therm. Fluid Sci. 66, 235 (2015)

9. Seghir-Ouali, S., Saury, D., Harmand, S., Phillipart, O., Laloy, D.: Convective heat transfer inside a rotating cylinder with an axial air flow. Int. J. Therm. Sci. 45, 1166 (2006)

10. Eriksson, D., Sundén, B.: Numerical investigation of the transient conduction in a rotating cylindrical shell exposed to an incident time varying heat flux. Int. J. Numer. Methods Heat Fluid Flow 6(6), 25 (1996)
11. Lucchi, M., Lorenzini, M., Valdiserri, P.: Energy performance of a ventilation system for a block of apartments with a ground source heat pump as generation system. J. Phys. Conf. Ser. 796(1), 012034 (2017)

12. Lucchi, M., Lorenzini, M.: Transient analysis of the radiative heating of rotating PVC pipes in a oven for end-forming process. Appl. Therm. Eng. 129, 84 (2018)

13. Heraeus group catalog: Infrared emitters for industrial processes. https://apps.heraeus.com/IR_Products_EN/mobile/index.html $\# p=1$. Accessed 16 Jan 2018

14. Yu, J., Sun, L., Ma, C., Qiao, Y., Yao, H.: Thermal degradation of PVC: a review. Waste Manag. 48, 300 (2016)

15. Howell, J., Siegel, R., Menguc, M.P.: Thermal Radiation Heat Transfer. CRC Press - Taylor \& Francis Group, Boca Raton (2010)

16. Shampine, L.: Vectorized adaptive quadrature in MATLAB. J. Comput. Appl. Math. 211, 131 (2008)

17. Shampine, L.: Matlab program for quadrature in 2D. Appl. Math. Comput. 202, 266 (2008)

18. Ahmad, W., Syed, K., Ishaq, M., Hassan, A., Iqbal, Z.: Numerical study of conjugate heat transfer in a double-pipe with exponential fins using DGFEM. Appl. Therm. Eng. 111, 1184 (2017)

19. Aneesh, A., Sharma, A., Srivastava, A., Vyas, K., Chaudhuri, P.: Thermal-hydraulic characteristics and performance of 3D straight channel based printed circuit heat exchanger. Appl. Therm. Eng. 98, 474 (2016)

20. Henning, T., Brandner, J., Schubert, K., Lorenzini, M., Morini, G.: Low-frequency instabilities in the operation of metallic multimicrochannel evaporators. Heat Transf. Eng. 28, 834 (2007)

21. Morini, G., Yang, Y., Lorenzini, M.: Experimental analysis of gas micro-convection through commercial microtubes. Exp. Heat Transf. 25, 151 (2012)

22. Yang, Y., Chalabi, H., Lorenzini, M., Morini, G.: The effect on the Nusselt number of the nonlinear axial temperature distribution of gas flows through microtubes. Heat Transf. Eng. 35, 159 (2014)

23. Etemad, G.A.: Free convection heat transfer from a rotating horizontal cylinder to ambient air: with interferometric study of flow. Trans. ASME 77, 1283 (1955)

Publisher's Note Springer Nature remains neutral with regard to jurisdictional claims in published maps and institutional affiliations. 